\title{
Scaling Laws in the Cosmic Structure and Renormalization Group
}

\author{
José Gaite $\dagger$ and Alvaro Domínguez $\ddagger$ \\ $\dagger$ IMAFF, CSIC, Serrano 113 bis, E-28006 Madrid, Spain \\ ‡Física Teórica, Univ. Sevilla, Apdo. 1065, E-41080, Sevilla, Spain
}

\begin{abstract}
There is evidence of a scale-invariant matter distribution up to scales over 10 Megaparsecs. We review scaling (fractal or multifractal) models of large scale structure and their observational evidence. We conclude that the dynamics of cosmological structure formation seems to be driven to a multifractal attractor. This supports previous studies, which we review, of structure formation by means of the renormalization group within a hydrodynamic formulation.
\end{abstract}

\section{Introduction}

Both the old idea of hierarchical clustering in the Universe and the statistical analysis of the distribution of galaxies have led to scaling laws in the cosmic structure and fractal models of it [1]. Scaling is usually demonstrated by the appearance of power laws in the correlation functions. There are other scaling laws in the cosmic structure, referring to other features. For example, the counterpart of galaxy clusters are galaxy voids, namely, large empty regions in the galaxy distribution. Fractal voids have scaling properties in the rank-ordering of voids [2], verified in galaxy surveys [3].

Nevertheless, a consensus on the range of application of scaling laws in the description of the cosmic structure has not been reached (a recent discussion is in [4]). The most general scaling model is the multifractal model, introduced in cosmology to describe "non-uniform" fractal clustering [5]. However, the study of cosmological $N$-body simulations has led to halo models of large scale structure [6] which do not assume any scaling laws. Fortunately, multifractal models can be formulated in a way that closely resembles usual halo models, allowing us to unveil scaling laws in $N$-body simulations [7].

Further to the description of cosmic structure, the problem of structure formation has given rise to scaling laws with dynamical content. For example, a popular model of structure formation, the adhesion model [8, 9], displays dynamical scaling and, in addition, leads to multifractal structure [10]. Structure formation is a nonlinear process, like other non-equilibrium processes in statistical mechanics that have scale invariance. Scaling is crucial in critical phenomena, which are phenomena that take place in equilibrium statistical mechanics for definite values of the parameters. The renormalization group (RG), which arose in quantum field theory, was soon applied to critical phenomena [11] and, more recently, it has also been applied to nonequilibrium processes ("dynamical RG"). The latter application can be extended to the process of structure formation in cosmology. However, the dynamical RG approach is perturbative and encounters some problems in this regard. 
A basic feature of modern formulations of the RG is that they deal with manybody systems by progressively removing irrelevant degrees of freedom until the "renormalized system" becomes manageable. The coarse-graining procedure, widely used in statistical mechanics and hydrodynamics, is inspired in the same idea. It is intrinsically non-perturbative and can be applied to structure formation.

We begin by reviewing evidence of scaling in the large scale structure of matter, arising from observational data or cosmological $N$-body simulations. Our analysis is consistent with the hypothesis that the dynamics of structure formation is driven to a multifractal attractor, like other non-equilibrium processes in statistical mechanics. This result justifies the hypothesis of dynamical scaling and, in particular, the dynamical RG approach. We connect with work along this line within the adhesion model, which is quite succesful in one dimension, but seems to require additional ingredients in three dimensions. Therefore, we turn to approaches based on the coarsegraining procedure and in particular, we formulate the coarse-graining "exact" RG group for structure formation.

Since we assume the existence of a multifractal attractor, we will not review approaches based on perturbation theory about the linear regime. Here we just mention the interesting recent application of the RG by Crocce \& Scoccimarro [12].

\section{Scaling laws in the large scale structure}

\subsection{Scaling of galaxy clustering}

Hierarchical clustering consists of a hierarchy of clusters of clusters. In general, fractal geometry studies sets (or functions) that are irregular (non-smooth) and have fine structure, namely, detail at all scales. Usually, the fine structure of a fractal is due to its self-similarity, that is, to the set being similar to parts of itself, in a strict or approximate sense. Random fractals only have statistical self-similarity, which implies that the correlation functions are power laws.

A useful description of random fractals is through the number-radius relation, which expresses the number of points in a ball of radius $r$ centered on one point and averaged over every point: it has to be the power law $N(r)=B r^{D}$, where $D$ is the fractal dimension and $B$ a constant. $N(r)$ is the cumulative conditional probability, that is, the integral of the conditional probability $\Gamma(r)$, which measures the average probability of finding another point at distance $r$ from one given point. In turn, $\Gamma(r)$ is directly related to the reduced two-point correlation function $\xi(r)$, namely, $\Gamma(r)=\bar{\rho}(1+\xi(r))$, where $\bar{\rho}$ is the average density. Both $N(r)$ and $\Gamma(r)$ are used to test scaling: their log-log plots must be linear, with slopes, $D$ and $D-3$, respectively.

The distribution must have a transition to homogeneity on very large scales, where $D \rightarrow 3$. The corresponding scale can be defined in terms of the non-dimensional correlation $\xi(r)$, by writing it as $\xi(r)=\left(r_{0} / r\right)^{\gamma}$, where $\gamma=3-D$ and $r_{0}$ is the scale of transition to homogeneity. We see that the strongly non-linear regime, $\xi \gg 1$, is the fractal regime, where $\xi, \Gamma$ and $N$ are all power laws. In the homogeneous regime, for $r \gg r_{0}, \xi \rightarrow 0$ and $\Gamma \rightarrow \bar{\rho}$, while $N \propto r^{3}$.

Typical values of the fractal dimension and the scale of transition to homogeneity are about $D \simeq 2[1,13]$ and $r_{0} \simeq 15 \mathrm{Mpc}^{-1}$ [13], respectively.

2.1.1. Scaling of voids Voids in the galaxy distribution scale if the number of voids with a given size is a power law of the size. It is again convenient to employ the 
cumulative count, namely, the number of voids $N(L>\ell)$ with linear size larger than a reference $\ell$, which fulfills $N(L>\ell) \propto \ell^{-D}$, where $D$ is the fractal dimension [2]. The cumulative count is the rank, so the preceding law can also be expressed as a powerlaw dependence of size with rank (if size refers to volume, the exponent is $3 / D$ ). Such type of dependence is an instance of Zipf's law.

The scaling of voids in galaxy surveys is still uncertain. Recent analyses find sets of convex-like voids that satisfy Zipf's law [3]. The fractal dimension deduced from them, $D \simeq 2$, coincides with the previously cited dimension deduced from clustering. However, $D=2$ is the (box-counting) dimension of the boundary of voids and, therefore, the minimal value of $D$ in the Zipf law for voids [14]. The actual fractal dimensions of the samples may be smaller [3].

2.1.2. Luminosity segregation Different galaxy populations may have different statistical properties. If these populations are fractal, they may have different dimensions. In fact, although $D \simeq 2$ is typical, other analyses yield smaller values, and one can change $D$ somewhat by selecting different galaxy populations: a systematic analysis of galaxy populations in the Sloan Digital Sky Survey (SDSS) selected by luminosity, made by M. Montuori [15], shows a decrease of fractal dimension with luminosity. Thus, the galaxy distribution may be, rather than a simple fractal, a multifractal, in which various dimensions appear naturally.

\subsection{Multifractal model}

Multifractals are the most general scaling distributions. They appear frequently as attractors of nonlinear dynamical systems. Multifractal measures represent highly irregular mass distributions, that is, with mass concentrations of very different magnitude. This magnitude is defined by the local dimension $\alpha(x)$ :

$$
m[B(\boldsymbol{x}, r)] \sim r^{\alpha(\boldsymbol{x})},
$$

where $m[B(\boldsymbol{x}, r)]$ is the mass in the ball of radius $r$ centered on $x$. In a regular mass distribution, $\alpha=3$ (constant), so mass concentrations $\alpha(\boldsymbol{x})<3$ are singularities. On the other hand, an ordinary fractal can be considered endowed with a uniform mass distribution over it, such that $\alpha<3$ is the constant fractal dimension. Thus, in the context of multifractals, ordinary fractals are called monofractals (or unifractals). Fullfledged multifractals possess a range of dimensions $\alpha$, namely, $0<\alpha_{\min } \leq \alpha \leq \alpha_{\max }$. Every set of points in which $\alpha$ takes a definite value is a fractal set. Therefore, a multifractal can be considered as a set of interwoven fractals with running $\alpha$. The multifractal spectrum $f(\alpha)$ is the function that gives the dimension of the fractal with exponent $\alpha$.

Statistical moments are defined by

$$
M_{q}(r)=\int d m(\boldsymbol{x}) m[B(\boldsymbol{x}, r)]^{q-1} .
$$

$M_{1}$ is the total mass (normalized to one). The two-point correlation integral $M_{2}(r)$ is the continuous version of the number function $N(r)$. Multifractals are singular non-uniform distributions, so moments with integer $q$ are not sufficient; one has to consider the full set of moments $M_{q}(r)$ for $-\infty<q<\infty$. We can then define the function $\tau(q)$ that gives the scaling behaviour of this full set of moments,

$$
M_{q}(r) \sim r^{\tau(q)} .
$$


$\tau(q)$ determines the multifractal spectrum through a Legendre transform [5]: assuming $\alpha(q)=\tau^{\prime}(q)$ to be monotone, $f(\alpha)=q(\alpha) \alpha-\tau[q(\alpha)]$.

2.2.1. The fractal distribution of halos We associate halos with singular mass concentrations, namely, points with $\alpha(x)<3$, such that the density given by Eq. (1) diverges as $r \rightarrow 0$. Note that scale invariance prevents us from assigning these singularities definite sizes or masses. Therefore, to properly define halos we must use some small coarse-graining scale $L$. In $N$-body simulations, the natural coarsegraining scale is the linear size of the volume per particle. Initially and during the linear evolution, there is one particle per volume element. So halos only arise in the nonlinear stage, as some volume elements concentrate particles from other regions that become voids.

Therefore, we identify halos with mass concentrations of size $L$ in a multifractal. Since $\alpha \sim \log m / \log L$, every population formed by equal-mass halos is a monofractal, although different populations have different dimensions. We can describe this difference between populations as a kind of bias, albeit of non-linear type. In a multifractal analysis of $N$-body simulations [7], we have found that populations of halos of given mass are fractals, with mass-dependent dimension. Their common scale of transition to homogeneity is $r_{0} \simeq 14 h^{-1} \mathrm{Mpc}$.

An interesting quantity is the mass function of halos, namely, the number of halos with a given number of particles. The Press-Schechter formalism predicts a power law (exponentially cut off at large mass) with exponent connected with the initial power spectrum [6]. This form is observed in our analysis, but the power law has a fixed exponent, namely, $N(m) \sim m^{-2}$, which corresponds to the spectral index $n=-3$ of the initial power spectrum, just beyond the allowed range. Moreover, the initial power spectra of the simulations we have analysed are not power laws, but the mass funtion is always the same, independently of the initial conditions.

\section{Dynamical scaling}

In the strongly nonlinear regime, when the initial condition is forgotten, dynamical scaling implies that a dynamical field $\varphi(\boldsymbol{x}, t)$ satisfies a scaling relation $\varphi_{L}-\langle\varphi\rangle \sim$ $L^{\chi} f\left(t / L^{z}\right)$, where $\varphi_{L}$ is the field coarse-grained over a length $L$ (see Eq. (7)), $\chi, z$ are critical exponents, $\lim _{u \rightarrow \infty} f(u)=1$ and $\lim _{u \rightarrow 0} f(u) \sim u^{\chi / z}$. In words, the fluctuations grow with time as one power law and they eventually reach saturation, in which state they depend on $L$ as another power law. Dynamical scaling is customary in the physics of surface growth and other non-equilibrium processes [16]. Similarly to the situation in static critical phenomena, the possible types of critical dynamics correspond to (attractive) fixed points of the dynamical RG. This tool allows one to compute the exponents $\chi$ and $z$ exactly or approximately.

\subsection{The adhesion model}

In the cosmological context, the linear regime is identified with small departures from the homogeneus Hubble expansion. The linear dynamics is described by a set of linearized equations for density and velocity. The phenomenological adhesion model is the simplest dynamics describing nonlinear structure formation in cosmology. It relies on the Zeldovich approximation [9], which consists in extrapolating into the nonlinear regime the condition of parallelism between velocity and gravity arising in the linear 
regime. In terms of a rescaled time and a rescaled velocity measuring departures from the Hubble flow, the adhesion model reduces to the Burgers equation (originally, an equation for compressible turbulence) $[8,10]$ :

$$
\frac{\partial \boldsymbol{u}}{\partial t}+\boldsymbol{u} \cdot \nabla \boldsymbol{u}=\nu \nabla^{2} \boldsymbol{u}
$$

with $\nabla \times \boldsymbol{u}=\mathbf{0}$ by the parallelism assumption, and the mass distribution is obtained from the field $\boldsymbol{u}(\boldsymbol{r}, t)$ by means of the continuity equation. Here, $\nu \rightarrow 0^{+}$is a phenomenological vanishingly small viscosity modelling the coupling to the unresolved small-scale degrees of freedom. The case $\nu=0$ corresponds actually to the Zel'dovich approximation: the fluid elements move with constant velocity (in the rescaled variables) along the initial gravitational acceleration, thus effectively neglecting the effect of pressure, viscosity or other small scale effects. Obviously, singularities arise and the density field diverges. A small, but nonvanishing value of $\nu$ regularizes these singularities into shocks: it amounts to an inelastic collision prescription, such that fluid particles adhere to each other at caustics, which become the walls ("pancakes"), filaments and nodes that are typical of large scale structure.

In the one-dimensional case, parallelism is exact. With scale-invariant initial conditions [10], the mass concentrates in shocks located in a dense set $\S$, at which $\boldsymbol{u}$ has discontinuities. This distribution is actually multifractal, namely, a peculiar type of bifractal. Furthermore, this bifractal evolves in time: the large shocks grow at the expense of smaller ones, illustrating the bottom-up structure formation typical of cold dark matter.

In three dimensions, the walls, filaments and nodes are definite lower dimensional objects, expected to arise in a generic situation. Therefore, a naive picture of this structure consists of a distribution of one, two and three-dimensional objects, that is, a trivial example of multifractal distribution, with integer-dimension objects only. However, as the initial velocity field is a non-smooth Gaussian random field [10], the structure produced resembles a self-similar distribution of walls, filaments and nodes that has been dubbed the cosmic web. In this "web" the mass concentrates, in addition to walls, filaments and nodes, in some regions rather than in others (because those objects concentrate there). The cosmic web is a non-trivial multifractal.

\subsection{Kardar-Parisi-Zhang equation}

The dissipative nature of the the Burgers equation (4) is directly related to the coupling to small scales. It seems natural to assume that the dissipation is complemented by a stochastic force (or noise). In terms of the velocity potential $\varphi$, i.e. $\boldsymbol{u}=-\nabla \varphi$, the stochastic Burgers equation becomes the Kardar-Parisi-Zhang equation\|,

$\frac{\partial \varphi}{\partial t}-\frac{1}{2}(\nabla \varphi)^{2}=\nu \nabla^{2} \varphi+\eta, \quad\left\langle\eta(\boldsymbol{x}, t) \eta\left(\boldsymbol{x}^{\prime}, t^{\prime}\right)\right\rangle=D \delta\left(\boldsymbol{x}-\boldsymbol{x}^{\prime}\right) \delta\left(t-t^{\prime}\right)$.

The KPZ equation has critical regimes where $\varphi$ exhibits dynamical scaling and the matter distribution is no longer determined solely by the initial conditions, but is instead the outcome of the interplay between the noise and the other terms of the equation. As a general result, invariance under Galilean boosts required by the convective nonlinearity implies $\chi+z=2$.

$\ddagger$ The situation is analogous to the formation of caustics in geometric optics.

$\S$ The adjective "dense" is understood with its mathematical meaning: a set is dense in an interval, say, if in any sub-interval, however small, there are points of the set.

II Under this name, this equation is used for the description of surface growth. 
In the one-dimensional case, the large scale dynamics is dominated by a weakly nonlinear (i.e., perturbative) fixed point at which $\chi=1 / 2$ and $z=3 / 2$. Furthermore, the Fokker-Planck equation for the probability $\Pi[\varphi(x), t]$ associated to the KPZ equation (5) and its stationary solution are

$\frac{\partial \Pi}{\partial t}=-\int d x \frac{\delta}{\delta \varphi(x)}[F(\varphi) \Pi]+\frac{D}{2} \int d x \frac{\delta^{2} \Pi}{\delta \varphi(x)^{2}} \Rightarrow \Pi_{\text {stat }}=\exp \left(-\frac{\nu}{2 D} \int d x\left(\partial_{x} \varphi\right)^{2}\right)$,

where $F(\varphi)=\nu \partial_{x}^{2} \varphi+\frac{1}{2}\left(\partial_{x} \varphi\right)^{2} . \quad \Pi_{\text {stat }}$ is a Boltzmann velocity distribution at temperature $k T=2 D / \nu$. Although $\Pi_{\text {stat }}$ is Gaussian, as in the absence of nonlinearity, the value of $z$ is different from the linear-equation value $\left(z_{\text {lin }}=2\right.$ associated to the diffusion equation). In consequence, the dynamical scaling regime can be considered linear as regards the stationary distribution, but not as regards temporal scaling. Some features involving temporal scaling can be calculated perturbatively with the RG. According to the stationary solution, the shocks predicted by the adhesion model in $d=1$ disappear at large times as the noise kicks in at a finite temperature $T$.

The critical dimension of the KPZ equation (5) is $d=2$, meaning that the nonlinearity is relevant if $d \leq 2$. For $d>2$, the equation becomes perturbatively nonrenormalizable, but there is evidence of a scaling strong-coupling regime. In particular, there is evidence based on the non-perturbative RG [17], a tool that we will introduce in Sect. 4.2. In $d=3$, the KPZ equation driven by the more general coloured noise, $\left\langle\eta(\boldsymbol{x}, t) \eta\left(\boldsymbol{x}^{\prime}, t^{\prime}\right)\right\rangle \propto\left|\boldsymbol{x}-\boldsymbol{x}^{\prime}\right|^{2 \rho-3}$, has been considered in the cosmological context [18]. There appear weakly nonlinear fixed points with exponents $\chi, z$ depending sensitively on the decay exponent $\rho$ of the noise correlator.

A likely failure of the adhesion and KPZ approach is the absence of vorticity imposed by the assumption of parallelism between velocity and gravity. Recently, Antonov [19] applied the RG to the Burgers equation appended with a colored stochastic source of vorticity. A perturbative fixed point arises where the scaling behavior is related to the generation of vorticity and depends on the decay exponent of the noise.

\section{Approaches based on coarse graining}

The KPZ models are rather phenomenological; namely, they miss a first-principle derivation of or a physical argument for the choice of noise correlator, which would make a quantitative prediction available. Now we review briefly more systematic approaches, which are still under development.

\subsection{The Small-Size Expansion}

The hydrodynamic equations are macroscopic equations, following from microscopic Newtonian mechanics of particles through an averaging process called coarse graining. This idea is implemented with the help of a window function $W_{L}(\boldsymbol{r})$, that is, a function that quickly vanishes outside a neighborhood of the origin of size $L$; typical examples are the sharp-cutoff ("top-hat") window and the Gaussian window. Thus, the coarsegrained mass density field $\varrho_{L}(\boldsymbol{r})$ is defined as the convolution

$$
\varrho_{L}(\boldsymbol{r})=\int d \boldsymbol{x} W_{L}(\boldsymbol{r}-\boldsymbol{x}) \varrho(\boldsymbol{x})
$$

with the microscopic mass density field $\varrho(\boldsymbol{x})$. One defines similarly a velocity field $\boldsymbol{v}_{L}(\boldsymbol{r})$, etc. The exact set of equations for $\varrho_{L}$ and $\boldsymbol{v}_{L}$, corresponding to the balance 
of mass and momentum, contain unknown terms which describe the coupling of the coarse-grained dynamics to the degrees of freedom in scales $<L$. Thus, a constitutive relation is required expressing these terms as functions of the coarse-grained fields.

Recently, the "Small-Size Expansion" (SSE) has been proposed [20], which relies on the physical hypothesis that the main contribution by the small scales stems precisely from the scales close to $L$. One finds a gradient expansion like those suggested in the coarse-grain approach to incompressible turbulence. In particular, the phenomenological viscous term in the adhesion model (4) is replaced to lowest order in the expansion by a term proportional to

$$
\frac{L^{2}}{\varrho_{L}}\left\{\left(\nabla \varrho_{L} \cdot \nabla\right) \boldsymbol{g}_{L}-\nabla \cdot\left[\varrho_{L} \sum_{k}\left(\frac{\partial \boldsymbol{v}_{L}}{\partial x_{k}}\right)\left(\frac{\partial \boldsymbol{v}_{L}}{\partial x_{k}}\right)\right]\right\}
$$

where $\boldsymbol{g}_{L}$ is the coarse-grained gravitational field. This term may act like a drain of kinetic energy in collapsing regions, and the adhesion model can be actually recovered under stronger dynamical assumptions like parallelism of $\boldsymbol{v}_{L}$ and $\boldsymbol{g}_{L}$. However, unlike the adhesion model, this term also behaves as a source of vorticity.

\subsection{The exact renormalization group}

The coarse-grained variables change with the coarse-graining length $L$ and so does the probability distribution, e.g., of the density $\mathcal{P}_{L}[\varrho]$ (in Fourier space):

$\frac{\partial}{\partial L} \mathcal{P}_{L}[\varrho]=\frac{d \ln \tilde{W}_{L}^{2}}{d L} \frac{\delta}{\delta \varrho}\left(\varrho \mathcal{P}_{L}[\varrho]\right)+\frac{1}{2} \frac{d \tilde{W}_{L}^{2}}{d L} P(k) \frac{\delta^{2}}{\delta \varrho^{2}} \mathcal{P}_{L}[\varrho]$,

where $\tilde{W}_{L}(k)$ is the Fourier transform of the window function, defined in Eq. (7), and $P(k)$ is the large-scale power spectrum of the density fluctuations, i.e., the Fourier transform of the correlation $\xi(r)$ introduced in Sec. 2.1. The differential equation (9), describing evolution with the scale $L$, is the exact $R G$ equation for $\mathcal{P}_{L}$, which is a sort of Fokker-Planck equation for $L$-evolution [21].

The exact RG equation has been amply used in high-energy and statistical physics $[11,22]$, but it has not been studied in the astrophysical literature (see [21]). However, it has been noticed that the one-point density probability distribution $p_{L}(\varrho)$ satisfies a diffusion equation which can be used to find the Press-Schechter mass function of collapsed objects [23]. This diffusion equation is connected with Eq. (9).

4.2.1. Time evolution and $R G$ Peebles [24] noted that the temporal variable, in some cosmological solutions (scaling solutions), plays the role of a scaling parameter and proposed a type of renormalization in which the time evolution can be undone by a redefiniton of the space scale and the number and mass of particles. Therefore, Peebles assumes a relationship between time evolution and evolution under the change of scale, in the same fashion as dynamical scaling. That relationship admits a fuller formulation in terms of the exact RG: Eq. (9) can be compared with a Fokker-Planck equation for time evolution, like the one for the KPZ equation found in Sect. 3.2 [21].

\section{Conclusions}

From the observational standpoint, scaling in the large scale structure is well justified, but the measures are not sufficiently accurate yet to determine many details. The scale of homogeneity, which has been the subject of much controversy, seems to be in 
the range 10-20 Mpc/h. There seems to be no point in trying to determine a definite value of the fractal dimension. Rather, the distribution fits a multifractal, so its scaling properties are given by its multifractal spectrum, which can already be found with the help of $N$-body simulations [7]. The determination of fine morphological features requires further analysis of the galaxy distribution as well as $N$-body simulations, with appropriate tools such as statistical moments or more sophisticated tools (Minkowski functionals, etc.). Among these morphological features are the voids. Scaling of voids is beginning to be observed, but deeper studies of voids will depend on improvement on their definition and, hence, detection.

From the theoretical standpoint, scaling provides us with a handle in an otherwise almost intractable problem of nonlinear dynamics. We have seen that dynamical scaling is indeed sufficiently powerful to draw a convincing picture of structure formation in one-dimension, with the help of perturbation theory and the renormalization group. However, the realistic three-dimensional case may demand non-perturbative tools, which are rather complex and are still being developed.

\section{References}

[1] Sylos Labini F, Montuori M and Pietronero L 1998 Phys. Rep. 29361

[2] Gaite J and Manrubia S C 2002 Monthly Not. RAS 335977 Gaite J 2005 Eur. Phys. Jour. B 4793

[3] Tikhonov A V and Karachentsev I D, preprint astro-ph/0609109, to appear in Astrophys. J. Tikhonov A V, preprint astro-ph/0610689

[4] Jones B J, Martínez V J, Saar E and Trimble V 2004 Rev. Mod. Phys. 76, 1211

[5] Pietronero L 1987 Physica A $\mathbf{1 4 4} 257$ Jones B J, Martínez V J, Saar E and Einasto J 1988 Astrophys. J. 332 L1

[6] Cooray A and Sheth R 2002 Phys. Rep. 3721

[7] Gaite J 2005 Europhys. Lett. 71332

Gaite J, preprint astro-ph/0604202

[8] Gurbatov S N, Saichev A I and Shandarin S 1989 Monthly Not. RAS 236385

[9] Shandarin S F and Zeldovich Ya B 1989 Rev. Mod. Phys. 61185

[10] Vergassola M, Dubrulle B, Frisch U \& Noullez A 1994 Astron. Astrophys. 289325

[11] Wilson K G and Kogut J 1974 Phys. Rep. 12, 75

[12] Crocce M and Scoccimarro R 2006 Phys. Rev. D 73063519

[13] Tikhonov A V and Kopylov A I 2002 Astrophys. 4588

[14] Gaite J, preprint astro-ph/0603572, to appear in Physica D.

[15] Montuori M, Univ. of Rome report, unpublished

[16] Barabàsi A-L and Stanley H E, Fractal concepts in surface growth, Cambridge U.P. (1995)

[17] Canet L, preprint cond-mat/0509541

[18] Berera A and Fang L-Z 1994 Phys. Rev. Lett. 72458

Domínguez A, Hochberg D, Martín-García J M, Pérez-Mercader J and Schulman L S 1999 Astron. Astrophys. 344 27; Astron. Astrophys. 363373

Buchert T, Domínguez A and Pérez-Mercader J 1999 Astron. Astrophys. 349343

[19] Antonov N V 2004 Phys. Rev. Lett. 92161101

[20] Domínguez A 2000 Phys. Rev. D 62103501

Domínguez A 2002 Monthly Not. RAS 334435

Buchert T and Domínguez A 2005 Astron. Astrophys. 438443

Knebe A, Domínguez A and Domínguez-Tenreiro R 2006 Monthly Not. RAS 3711959

[21] Gaite J 2001 Int. J. Modern Phys. A 162041

[22] Morris T 1994 Int. J. Modern Phys. A 92411

Bagnuls C and Bervillier C 2001 Phys. Rep. 348, 91

[23] Bond J R, Cole S, Efstathiou G and Kaiser N 1991 Astrophys. J. 379, 440

[24] Peebles P J E 1985 Astrophys. J. 297350 